\title{
Development and deployment of distributed e-VLBI components
}

\author{
Karina Krinkele* \\ Researcher, Ventspils International Radio Astronomy Centre of Ventspils University College \\ (VIRAC), Latvia \\ E-mail: karina.krinkeleeventa.IV
}

Normunds Jekabsons

Senior researcher, Ventspils International Radio Astronomy Centre of Ventspils University College (VIRAC), Latvia

E-mail: normundsjeventa.Iv

Ivar Shmeld

Senior researcher, Ventspils International Radio Astronomy Centre of Ventspils University College (VIRAC), Latvia

E-mail: ivarsseventa.IV

\section{Mark Kettenis}

Software engineer, Joint Institute for VLBI in Europe (JIVE), The Netherlands

E-mail: kettenis@jive.nl

\section{Aard Keimpema}

Scientific programmer, Joint Institute for VLBI in Europe (JIVE), The Netherlands

E-mail: keimpema@jive.nl

\begin{abstract}
Recent trend in radio astronomical data processing is towards e-VLBI (Electronic Very Long Baseline Interferometry), which has several advantages to the more traditional delayed VLBI data processing. Under the ongoing EU FP7 NEXPReS project (Novel EXplorations Pushing Robust e-VLBI Services, 2010-2013) prototype of real-time data distributed processing system based on HPC (High performance computing) solutions for EVN (European VLBI Network) is created, effectively replacing previous Grid technology based astronomic data processing paradigm. In this paper some of software components developed for e-VLBI will be described, as well as the main reasons for the switch-over.
\end{abstract}

EGI Community Forum 2012 / EMI Second Technical Conference,

26-30 March, 2012

Munich, Germany

\footnotetext{
* Speaker.
} 


\section{Introduction}

VLBI (Very Long Baseline Interferometry) [4] is a method to observe astronomical objects (such as pulsars, quasars, black holes, etc.) with multiple radio telescopes simultaneously. Application of VLBI techniques allows to emulate a telescope with an aperture size comparable to the separation between the individual telescopes, sometimes reaching $1000 \mathrm{~km}$ and more. As a consequence, VLBI is one of the most accurate methods to do an astronomical observations.

Numerical data processing in the case of VLBI observations is mandatory. The main processing routine involves calculation of correlation function spectra for each pair of participant telescopes. High sampling rates at each station allows high sensitivity and better random noise elimination, altogether leading to substantial numerical complexity of the raw data processing.

More than 25 radio telescopes in Eastern and Western Europe, Russia, China and South Africa participates in EVN observations. Data processing center in JIVE takes care of data processing of the bulk of EVN data. Specially designed hardware - the EVN MkIV correlator, still is a main data processor there, although it is about to be replaced by the software based correlator (SFXC). Most data from radio telescopes are shipped to the correlator on it's physical media (disk packs). Motivation of such a time consuming procedure is the rather large amount of raw VLBI data combined with slow and sometimes unreliable Internet connections to the telescopes.

However, the substantial progress of data communication network quality and bandwidth increase reached during the last decades, makes electronic VLBI data transfer feasible. It seems that electronic data streaming technology, can be of daily use in a rather near future. This shift from transport of physical data media (which in some cases take several weeks) to on-line streaming gives new exciting opportunities to radio astronomers - from instant quality control and automation of observation sessions to observed data driven session flow.

A new data delivery method allows us to reconsider all data processing route. The new method is called e-VLBI or electronic Very Long Baseline Interferometry. The main traits of e-VLBI are: 1) data are streamed to the correlation center; 2) often for data correlation software data processor is used, which gives more flexibility by respect to numerical methods and hardware; 3) results are promptly sent back to a radio astronomers still during the same session.

Thus, radio astronomers can in-situ control and monitor an entire experiment. To make this easier there is an opportunity to automate the e-VLBI processes by using developed software components.

Subset of EVN network participants already can process data in a shared e-VLBI infrastructure developed by EU FP7 EXPReS project (Express Production Real-time e-VLBI Service, 20062009). The main outcomes [1] of EXPReS was introduction of basic e-VLBI components (the Correlator Node, the VLBI Broker, Translation Nodes and WorkFlow manager), as well as first deployment of a test system. The data transfer was split up in so called data chunks. After reception on the correlator side these chunks were temporally queued for following cross-correlation on storage of the HPC cluster.

Since July 2010, several institutions of EVN network participates in NEXPReS project with the ultimate goal of incorporating e-VLBI into every astronomical observation conducted by the EVN. This is an ongoing project, which pushes e-VLBI technologies further in the direction of real-time data processing, leading to several major changes in the related software. 


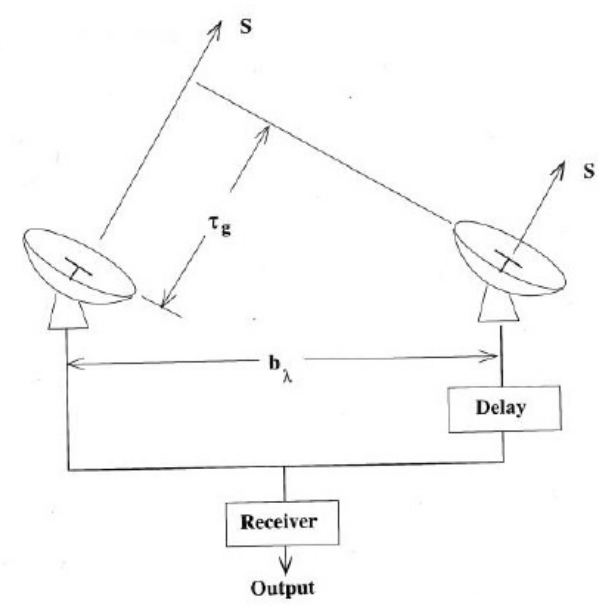

Figure 1: Interferometer scheme with two radio telescopes: $b_{\lambda}$ - base line, $\mathrm{S}$ - signal of radio source, $\tau-$ geometric time delay [4].

On the data transport level the chunking process is replaced with real-time streaming, the change which should eliminate the time delays of several minutes, observed in the previous eVLBI implementation.

On the workflow planning level the interaction with an operator (radio astronomer) is reworked by using modern WEB technologies. Much more roles for an experiment participants are introduced in the new system

Finally, upcoming e-VLBI implementation is engineered to be compatible with distributed correlation sessions in several correlation centres. Thus, single point of failure problem of both HPC facilities and network infrastructure can be avoided. It is worth to notice that data processing now can be performed at the correlation facilities of organization interested in observation results of particular e-VLBI session. Observation specific data processing routes this way can be maintained.

In the next sections authors described basics of VLBI observations, an e-VLBI system development and it's components, a general basis of distributed data processing in EVN observations and occurred tests of e-VLBI system in real-time.

\section{Basics of VLBI observations}

Radio interferometry is an accurate measurement method to establish set of small physical changes. The method is based on measurement of path difference of approaching electromagnetic wave fronts, which can be established by cross-correlation between two received distant signals from the same source. The basic elements of a two-element interferometer are shown in Figure 1.

The interferometer consists of two distant radio telescopes observing radio source of interest simultaneously. Electromagnetic radiation reaches both radio telescope receivers at different time moments, thus time delay compensation $\tau$ between telescopes is required: $\tau=\frac{\mathbf{b} \mathbf{s}}{c}$, where $\mathbf{s}$ is unit vector pointing to the radio source, $c=299782458(\mathrm{~m} / \mathrm{s})$ is the speed of light and $\mathbf{b}$ is the baseline vector [6]. The distance $D: D=\|\mathbf{b}\|$ between radio telescopes is called baseline. 


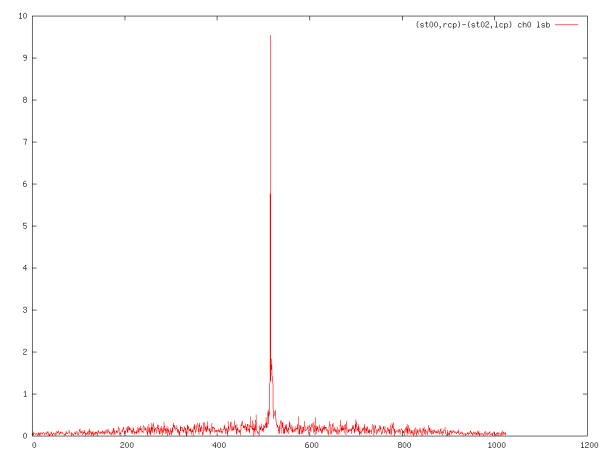

Figure 2: Fringe function - correlation between Irbene RT-32 radio telescope and Torun radio telescope, April 12, 2012.

Let's take a look on two signals: $g(t+\tau)$ and $h(t)$ received by respective radio telescopes. The cross-correlation of both signals: $\Gamma(h(t), g(t+\tau))$ is defined as

$$
\Gamma(h(t), g(t+\tau))=\int h^{*}(t) g(t+\tau) d \tau,
$$

where $f^{*}$ denotes the complex conjugate of $f$ [2].

For efficient computation of correlation function the correlation theorem [10] can be used. This way cross-product of Fourier transformed signals first is constructed in the frequency domain. Inverse transform yields $\Gamma$ in the time domain. Fast Fourier Transformation (FFT) algorithm can reduce overall algorithmic complexity to $O(N \log (N))$ operations.

There is an explicit maximum of correlation function by respect to the time delay $\tau, \tau_{m}$, related to the wave front path difference $\mathbf{b} \cdot \mathbf{s}=c \tau_{m}$.

With the aid of simple trigonometric conversations so called fringe function [2] (see at Figure 2.) can be constructed, from which an accurate angular position of observed radio source is obtained. Theoretically three VLBI stations are required to measure both celestial angles, however in practice more stations are needed for cancellation of numerous measurement errors and to produce the detailed radio map of object observed.

Angular resolution of emulated telescope can estimated as: $R \approx \frac{\lambda}{D}$ where $R$ is an angular resolution, and $\lambda$ is wavelength. So, as larger is baseline, as better is an angular resolution.

\section{3. e-VLBI development in EVN}

The long term goal set by EU FP6 EXPReS project was to create a real-time frame work for distributed correlation. As a first step towards this goal an off-line distributed correlation frame work was produced intended for pre-recorded data.

The architecture of the EXPReS frame work was very similar to that presented in this work. The main difference is that data was split into chunks, where each chunk represents several seconds of data. These chunks were then transferred to the worker nodes through grid-ftp. The worker processes in turn were scheduled using the local job-scheduling software. Unfortunately, latency of test system was rather high for e-VLBI. 
In NEXPReS project the goal is to build a real-time distributed correlator. The real-time aspect requires that there is guaranteed number of computational resources available, at low latency. An important aspect here is the network bandwidth, the current state of the art is $1 \mathrm{Gbit} / \mathrm{s}$ of data per station. Furthermore, the EVN is currently on an upgrade path to $4 \mathrm{Gbit} / \mathrm{s}$. A typical experiment consists of 10-16 stations. Guaranteeing such a bandwidth in a multi-user GRID environment is challenging.

Real-time grid architectures are currently an active field of research. When such technologies mature it would be advantageous to enable the distributed correlation platform to benefit from grid technologies. Especially the ability to dynamically add or remove computing resources on the fly over the course of an observation to match the current workload.

\subsection{Components}

The main components of e-VLBI system discussed are: Translation Nodes (location - radio telescopes), distributed software FX correlator (SFXC or DiFX, location - correlation centres), VLBI Broker, and WorkFlow manager (both - software modules, located anywhere), see Figure 3. Lets focus on more detail description of these.

\subsubsection{Radio telescopes}

Usually a single-dish radio telescope consists of a parabolic reflector which focuses incoming radio frequency energy on to a receiver input [9]. Later the signal is amplified, filtered, sampled and digitally split into frequency bands/channels. From e-VLBI point of view the output of the registration system is several byte stream (channels) each of which has to be cross-correlated with respective streams from an another sites. The MARK5A/B [11] and VDIF (MARK6) [12] are few of the most popular data formats of sampled data.

\subsubsection{Translation Nodes}

Translation Node (TN) is a software module implemented at each radio telescope on top of the signal recording system. TN is responsible for handling sampled data, which includes format conversions if any, and set up network data distribution using GridFTP protocol or via SCP. Recently observed data stream is buffered and migrated in small portions (data chunks). This feature will be replaced with continues streaming of VDIF data later on.

The list of TN related operations:

- Before the experiment starts, a radio astronomer defines the data flow from the radio telescopes to the distributed software correlator;

- Each TN is informed about the new experiment;

- TN splits received data into the packets and sets up data transfer connections to the distributed correlator nodes;

- Number of data packets are sent from radio telescope to the correlator;

- After acknowledgment of successful correlation from the distributed correlator TN deletes all data processed. If there is an error, $\mathrm{TN}$ re-sends non-correlated data again to the correlator. 


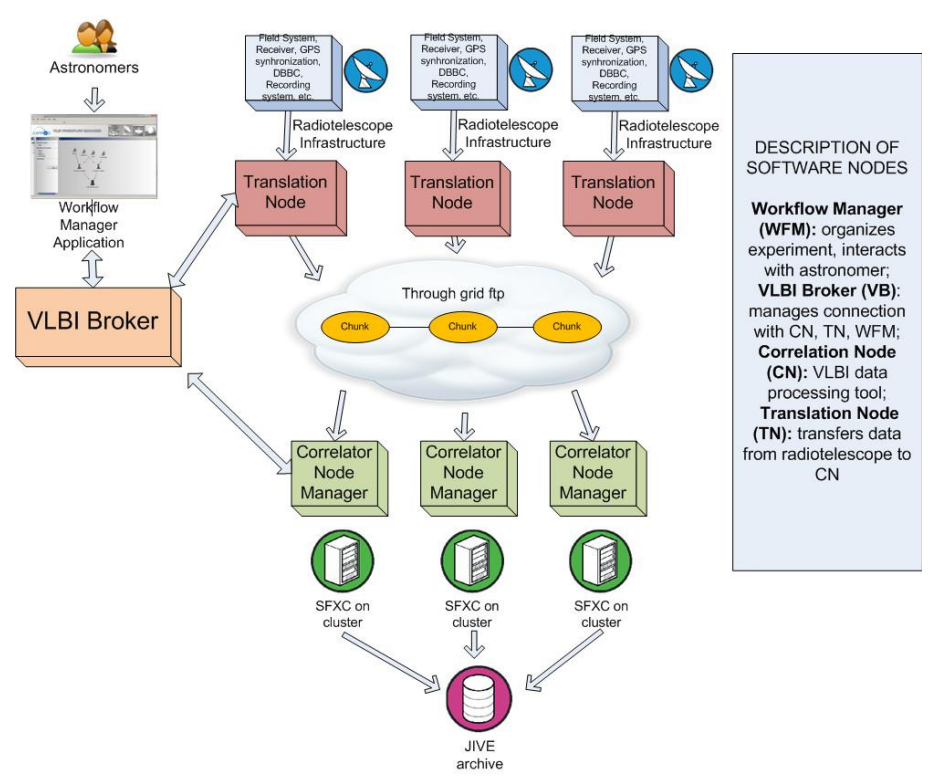

Figure 3: Automatic e-VLBI system in NEXPReS project.

\subsubsection{Correlation Nodes}

Each computational resource represents a separate, independent distributed software correlator module called Correlation Node $(\mathrm{CN})$ which can be used to correlate VLBI data. At the moment there are three CNs deployed - at High Performance Computers - at VIRAC, JIVE and PSNC (Poznan Supercomputing and Network Centre). The CN consists of two parts: VLBI data processing tool - Software FX correlator and correlation Node manager.

VLBI data correlator. During EXPReS project observational data processing tool called software FX correlator (SFXC) was deployed by JIVE. SFXC is developed mainly in $\mathrm{C}++$, and is distributed as open source. The correlator has to be executed on a high performance POSIX compatible computer cluster, where it utilizes parallel programming technologies based on Message Passing Interface (MPI) protocol. Notable SFXC traits are support of real-time data correlation as well as support of cross-correlation (the correlation of a signal from one radio telescope with a signal from another radio telescope) and autocorrelation (the cross-correlation of a signal with itself) for both - far objects of visible Universe together with much closer signal sources like space crafts inside the Solar system. Although SFXC is considered to be the default correlator for the EVN network, the overall e-VLBI approach allows use of other correlators, such as DiFX which is popular elsewhere.

Correlation Node manager (CNM) is responsible for managing and executing correlator (adapted for SFXC) jobs on the HPC cluster. CNM starts and stops the correlator on computer facilities, gets the correlation status information from the correlator and sends it back to a radio astronomer after data processing. At the end CNM sends correlated results to an archive.

\subsubsection{VLBI Broker}

VLBI Broker (VB) is the glue element of the e-VLBI System. It provides the automated 


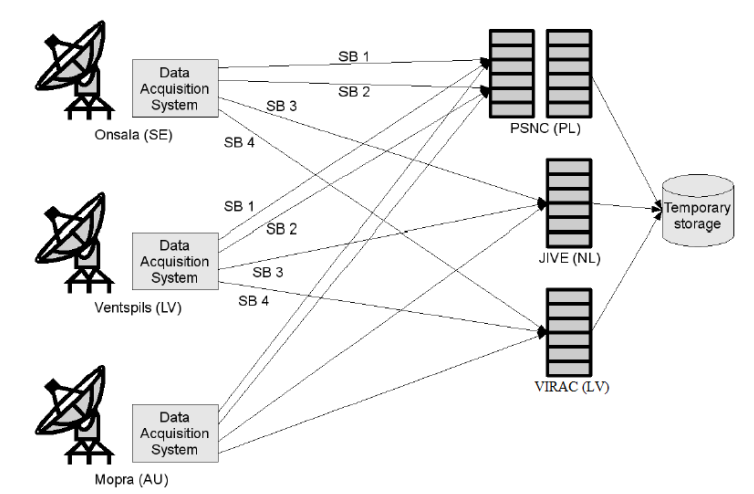

Figure 4: An example of a small e-VLBI sub-segment of distributed data processing.

control of the entire experiment. The plan of the experiment as a bundle of ASCII files of different formats has to be submitted by the radio astronomer before the observation starts. VB is responsible for coordination of tasks on Correlation and Translation Nodes.

During the experiment:

- A radio astronomer informs VB about the new experiment;

- VB manages communication with radio telescopes(TNs) and computational resources (CNMs);

- VB gets status information from CNMs and represents them.

\subsubsection{Workflow manager}

For the VB to be able to do its job exact definitions of storage elements, data flows, computation resources, etc. are needed. This "session work plan" consists of rather complex mix of different data formats, poorly suitable for human reading. Workflow Manager (WFM) is the main interface between user and the automatic e-VLBI system. Using WFM the e-VLBI system allows a radio astronomer to plan, execute and monitor his observations. WFM main job is to make life easy for a radio astronomer during the observation process.

New workflow definition is required for each new e-VLBI experiment.

The main steps to start new experiment are - to create an experiment description (VEX file) using the SCHED application, to set an experiment control parameters, to set all resource elements (TNs and CNs) and to inform VB about the new experiment with all necessary parameters. The first version of the WFM was deployed as a stand-alone Java application at PSNC during the EXPReS project. VIRAC and PSNC are developing the new WFM based on open source web platform Liferay.

\section{Distributed data processing}

In recent e-VLBI system data processing is distributed over multiple high performance computational resources. Consider a small e-VLBI sub-segment of distributed data processing, shown in Figure 4. 
At each radio telescope the VLBI data stream is split up in several streams, each containing a single frequency band/channel. These streams are then distributed over the available correlation centres. Since each frequency band can be processed independently of the other frequency bands, these streams can be processed in parallel. This way, distributed correlation minimizes total data transport, and avoids "bottlenecks" in the data transport.

Large number of radio telescope pairs (i.e. $0.5 K(K-1)$ ) with high sample rates (a few Gbits/s) and combined with minimum time intervals (a few seconds or more) for week signal accumulation to be detected generates a huge data amount which has to be processed (correlated). Thus, requirements on computational resources in correlation center for correlation of VLBI data is high. The good news are - each pair of radio telescopes can be correlated separately, thus efficient parallel algorithms for cluster or grid computing can be developed. Due to historical VLBI data processing evolution from single correlation point, the upcoming EVN e-VLBI implementation is designed for distributed cluster computing.

At the moment exists three distributed correlation node installations - in VIRAC, JIVE and PSNC.

\section{Tests of distributed e-VLBI system}

Two e-VLBI experiments was performed at April and May, year 2012.

The goal of both was to measure the performance of HPC cluster based distributed VLBI data correlation in real-time and to evaluate the completeness of developed components of e-VLBI system. Five radio telescopes from EVN were able to join the tests: Onsala, Torun, Yebes, Westerbork and Hartebeesthoek. HPC facilities at JIVE and PSNC were used for data processing. Data gathered by telescopes were sent for on-line correlation.

The tests were configured to split VLBI data in the domain of time, instead of domain of frequency, which is still under development. Data flow from each station had $128 \mathrm{MHz}$ spectrum, totally yielding data flow of $1 \mathrm{Gbit} / \mathrm{s}$. Within above mentioned automatic e-VLBI experiments the user was able to set up the correlation task within the application and execute the data processing using assigned distributed resources.

Generally tests were successful, which indicates that all operations related to correlation, data transport and recording were implemented correctly.

Although successful both tests revealed some minor configuration and runtime problems, foreseen before, for example, a temporary data storage problem in PSNC. Also, the data processing bottleneck of existing design was identified - it appears to be the speed of I/O operation in correlator software.

More performance tests using GPU (graphics processing unit) capability of the PSNC and VIRAC clusters are planned in the near future.

\section{Conclusions}

Distributed version of software correlation and an other e-VLBI components recently undergoes active development with an ultimate goal to fully automate VLBI observations - from session 
schedule to distribution of data flow and control of all data processing tasks. First EVN e-VLBI test sessions are already performed.

With reference to practical e-VLBI tests and experiments in EVN - high performance computing elements such as $+4 \mathrm{Gbit} / \mathrm{s}$ Internet now and $+10 \mathrm{Gbit} / \mathrm{s}$ in the future, cluster computing, etc. are mandatory for advancing e-VLBI technologies.

We are on a road towards to full e-VLBI distributed correlation system implementation per annum.

\section{ACKNOWLEDGMENTS}

Research leading to these results has received funding from the European Union's Seventh Framework Programme (FP7/2007-2013) under grant agreement no. RI-261525 NEXPReS. This material reflects only the authors' views, and the European Union is not liable for any use that may be made of the information contained therein.

The participation of the authors at EGI Community Forum 2012 / EMI Second Technical Conference was financed from ERDF's project

"SATTEH, No. 2010/0189/2DP/2.1.1.2.0/10/APIA/VIAA/019" being implemented in Engineering Research Institute "Ventspils International Radio Astronomy Centre" of Ventspils University College (VIRAC).

\section{References}

[1] L. Dolata, M. Okon, D.Stoklosa, Sz. Trocha, N. Meyer, M Stroinski, D. Kaliszan, T. Rajtar, M.Lawenda, User Interaction and Workflow Management in Grid enabled e-VLBI Experiments, Computational Methods in science and technology 15(1), 95-104 (2009)

[2] Romney J.D., Cross Correlators, Synthesis Imaging in Radio Astronomy II, ASP Conference Series, Vol 180, 57-78 (1999)

[3] Kettenis M., Keimpema A., Small D., Marchal D., e-VLBI with the SFXC correlator, in proceedings of The 8th International e-VLBI Workshop (2009)

[4] Bernard Burke F., Graham-Smith, An Introduction to Radio Astronomy, 2nd edition. Cambridge, UK, New York : Cambridge University Press, ISBN 0-521-80889-8 (2002)

[5] Okon, M., Stoklosa, D., Oerlemans, R., Langevelde, H.J. van, Kaliszan, D., Lawenda, M., Rajtar, T., Meyer, N., and Stroinski, M., Grid Integration of Future Arrays of Broadband Radio-Telescopes moving towards e-VLBI, Grid Enabled Remote Instrumentation. Springer, p. 571 (2008)

[6] Gordon D., MacMillan D., Baver. K, Calc 10 Implementation, IVS (International VLBI Service for Geodesy and Astronomy) General Meeting Proceedings (2006)

[7] Cambell R.M., e-VLBI and Other Developments at the EVN Data Processors at Jive Joint Institute for VLBI in Europe, 10th European VLBI Network Symposium and EVN Users Meeting: VLBI and the new generation of radio arrays (2010)

[8] Clark B.G., Coherence in Radio Astronomy, National Radio Astronomy Observatory, Published by the Astronomical Society of the Pacific, vol. 6.,ISBN 0-937707-23-6, eISBN 978-1-58381-342-3, $1-11(1989)$ 
[9] Wright A., Single-dish Radio Astronomy, Originally submitted for the Astronomy Online course at Swinburne University of Technology (2004)

[10] Brocewell, Ronald N., The Fourier Transform and its applications, Third Edition, McCrow-Hill Higer Education, ISBN 0-07-116043-4 (2000)

[11] Whitney A. R.,Mark 5 Disk-Based Gbps VLBI Data System, Massachusetts Institute of technology Haystack observatory (2004)

[12] Whitney A. R., Lapsley D. E., Taveniku M., Mark 6 VLBI data system; 16 Gbps recording demo, Massachusetts Institute of technology Haystack observatory (2011)

[13] N. Kruithof, E-VLBI using a software correlator, Grid Enabled Remote Instrumentation, Signals and Communication Technology, 537 - 545, ISBN 978-0-387-09662-9 (2009) 